\title{
Instrument to Measure Reflection on Participation in Quality Improvement Activities for Maintenance of Certification
}

1. This quality improvement project did not require much thought.

1 - Strongly disagree

2 - Disagree

3 - Neutral

4 - Agree

5 - Strongly agree

2. I was able to complete this quality improvement project without thinking about it.

1 - Strongly disagree

2 - Disagree

3 - Neutral

4 - Agree

5 - Strongly agree

3. When working on this quality improvement project, I did tasks without having to think about them.

1 - Strongly disagree

2 - Disagree

3 - Neutral

4 - Agree

5 - Strongly agree

4. When completing this quality improvement project, I continually reflected on what I had previously known about improvement methodologies.

1 - Strongly disagree

2 - Disagree

3 - Neutral

4 - Agree

5 - Strongly agree

5. I needed to understand the process of quality improvement to get credit for this project.

1 - Strongly disagree

2 - Disagree

3 - Neutral

4 - Agree

5 - Strongly agree 
6. Doing this project required me to understand the methodologies of quality improvement.

1 - Strongly disagree

2 - Disagree

3 - Neutral

4 - Agree

5 - Strongly agree

7. When doing this quality improvement project, I often reflected on my actions to see whether I could have improved on what I did.

1 - Strongly disagree

2 - Disagree

3 - Neutral

4 - Agree

5 - Strongly agree

8. After completing this quality improvement project, I considered what I had been doing and alternative ways of doing it.

1 - Strongly disagree

2 - Disagree

3 - Neutral

4 - Agree

5 - Strongly agree

9. Working on this quality improvement project caused me to question other peoples' habits, and consider ways to improve my own work.

1 - Strongly disagree

2 - Disagree

3 - Neutral

4 - Agree

5 - Strongly agree

10. As a result of completing this quality improvement project, I have changed my normal way of thinking about things.

1 - Strongly disagree

2 - Disagree

3 - Neutral

4 - Agree

5 - Strongly agree 
Supplemental Digital Appendix 1 for Wittich CQ, Reed DA, Ting HH, Berger RA, Nowicki KM, Blachman MJ, Mandrekar JN, Beckman TJ. Measuring Reflection on Participation in Quality Improvement Activities for Maintenance of Certification. Acad Med. 2014.

11. Doing this quality improvement project has challenged some of my firmly held ideas.

$$
\begin{aligned}
& 1 \text { - Strongly disagree } \\
& 2 \text { - Disagree } \\
& 3 \text { - Neutral } \\
& 4 \text { - Agree } \\
& 5 \text { - Strongly agree }
\end{aligned}
$$

12. When doing this quality improvement project, I discovered faults in what I had previously believed to be correct.

$$
\begin{aligned}
& 1 \text { - Strongly disagree } \\
& 2 \text { - Disagree } \\
& 3 \text { - Neutral } \\
& 4 \text { - Agree } \\
& 5 \text { - Strongly agree }
\end{aligned}
$$

